\title{
Cinco buenas prácticas para conformar una sociedad de aprendizaje servicio
}

\author{
Manuel Caire Espinoza mcaire@uahurtado.cl \\ Universidad Alberto Hurtado, Chile.
}

\section{Resumen}

Todo proyecto de aprendizaje servicio supone un campo dinámico de acción, donde difícilmente se pueden anticipar los resultados de este. No obstante, hay ciertos elementos que al menos se deben resguardar a la hora de planificar y ejecutar estos proyectos. Uno de esos elementos es la noción de sociedad de aprendizaje servicio. En el rol de asesor de estos proyectos, he conocido muchas experiencias que me han permitido configurar al menos cinco tópicos que se reiteran y que en este artículo se presentan como buenas prácticas para tener en consideración. Por cierto que ninguna de ella es más importante que otra. Tampoco significa que la ausencia de una o más perjudiquen la calidad de los proyectos. Están llamadas a abrir preguntas y ser un foco de atención a la hora de enfrentar un proyecto de aprendizaje servicio.

\section{Palabras clave}

Aprendizaje servicio, educación superior, comunidad, buenas prácticas, asesoramiento. 


\title{
Five Best Practices for Shaping a Learning-Service Society
}

\begin{abstract}
Every service-learning project involves a dynamic field of work, where the actual results can seldom be predicted. There are certain elements, however, that must be maintained when planning and delivering service-learning projects, one element being society's notion of service-learning. I have had many experiences which have helped me to identify at least five recurring topics. In this paper, these five topics are presented as best practices to take into consideration; no one is more important than another. The lack of one or more of the identified topics will hinder the quality of the projects. They should provoke questions and to serve as a focus of attention when facing any service-learning project.
\end{abstract}

\section{Keywords}

Learning-Service, Higher Education, Community, Best Practices, Advice. 


\section{Introducción}

Existe un creciente interés en el ámbito de la Educación Superior por generar prácticas innovadoras de enseñanza-aprendizaje centradas en el estudiante. De la mano con este interés, las universidades han ratificado en el último tiempo, la necesidad de incorporar explícitamente estrategias activas de aprendizaje en el curriculum de los estudiantes, donde se pongan a prueba las competencias profesionales en situaciones reales. Una de estas alternativas educativas es el aprendizaje servicio, un enfoque de enseñanza aprendizaje que involucra a estudiantes en el desarrollo de un servicio en la comunidad para alcanzar sus objetivos académicos (Furco y Billig, 2002). El término aprendizaje servicio se utiliza para denominar experiencias o programas específicos, desarrolladas por un grupo particular de jóvenes o adultos, en el contexto de instituciones educativas o de organizaciones sociales. Designa también una propuesta pedagógica y una forma específica de intervención social (Tapia, 2010, 26).

En Chile, las experiencias de aprendizaje servicio centran sus esfuerzos en desarrollar alianzas con comunidades, organizaciones y/o colectivos que se encuentran en situaciones de alta vulnerabilidad, con condiciones de exclusión social, asociadas a drogadicción, deserción escolar, discriminación social, abandono, entre otros. Esto trae consigo una decisión importante: aportar desde los conocimientos curriculares a resolver problemas o necesidades sociales. En mis seis años de experiencia profesional asesorando proyectos en esta materia, he visto más de 200 proyectos de aprendizaje servicio. Docentes, estudiantes y socios comunitarios trabajando juntos $-\mathrm{y}$ a veces no tanto- por afrontar problemáticas complejas. El trabajo de asesoramiento, dirán Andreozzi y Nicastro (2006) es un acto de intervención entendido como un venir entre, un interponerse $y$, en ese sentido, queda ligada a la acción de colaborar y acompañar en la producción de conocimiento y en el desarrollo de cambios personales, grupales $u$ organizacionales.

Pensar el asesor como un provocador que promueve, a través de un dispositivo de trabajo determinado, el surgimiento de aquello que se convertirá en objeto de análisis (Andreozzi y Nicastro, 2006, 40) es sin duda el motor que ha movido mi labor en estos años y que quisiera compartir en esta breve reflexión, a partir de un punto que parece sustancial a la hora de generar estos proyectos ¿cómo crear una sociedad de aprendizaje servicio donde todos los actores confluyan armónicamente para el logro de los objetivos propuestos? Pensar en las condiciones de posibilidad obliga a detenernos y reflexionar en aquellos puntos sensibles de cada proyecto.

Antes de responder esto, quisiera plantear tres interrogantes previos para la contextualización de lo que sería una sociedad de aprendizaje servicio. 


\section{Algunos interrogantes frente a este tema}

En primer lugar ¿qué y quién es el socio comunitario? ¿Qué o quién es la comunidad cuando nos referimos a este término? Es necesaria una mirada crítica sobre el concepto de comunidad en la retórica del aprendizaje servicio. Algunos identifican comunidad con el personal de la ONG y/o los directores de estos organismos. Otros interpretan comunidad a la gente de su barrio o de su localidad geográfica (Cruz y Giles, 2000). Para algunos docentes, es más pertinente que sean organizaciones formales, fundaciones u ONG con cierta estructura que permita trabajar de la mano con los equipos de intervención, es decir, profesionales o técnicos de estas instituciones. Pero para otras experiencias, el socio comunitario más idóneo es un microempresario, o un colectivo de personas migrantes. Este reconocimiento es importante hacerlo al momento de planificar un proyecto para reconocer a los actores que conformarán el trabajo.

Segundo ¿cómo asegurar contextos de servicio de calidad? Una investigación de Eyler y Giles (1999) mostró que los estudiantes valoraban significativamente aquellos proyectos de aprendizaje servicio donde tenían que ejecutar diversas tareas, asumían responsabilidades importantes y un trabajo interesante que les había dado lugar a una nueva perspectiva sobre los temas sociales. Tal vez, cuando planificamos los proyectos, un componente importante es que aquellos que coordinan las experiencias -desde la universidad y desde la comunidad- sean capaces de mensurar el desafío que esto implica. Sumado a esto, es necesario considerar otras variables que pueden incidir en la percepción de servicio de calidad. A veces con las mejores intenciones se planifica un proyecto en los lugares más alejados de nuestra ciudad. No obstante, los largos tiempos de traslado de los estudiantes pueden afectar la intensidad del trabajo. $\mathrm{O}$ a veces las comunidades solo pueden participar en horarios de fines de semana o por las tarde-noche y eso, también puede dificultar las labores. O bien, la cantidad de estudiantes es insuficiente para dar una buena respuesta a las demandas sociales. Estas cuestiones son importantes mencionarlas para no creer que esto es llegar y hacer.

Y tercero ¿qué papel juega un socio comunitario en una experiencia de aprendizaje servicio? Es bueno considerar la noción de aprendizaje servicio que tenga la comunidad. Esto ayudará a identificar los grados de participación en el proyecto. A mayor comprensión, mayores serán las opciones de participar en procesos de inducción con los estudiantes, espacios de reflexión, momentos de evaluación y retroalimentación. Una manera creativa de identificar el rol que tienen las comunidades es centrarse en los activos que poseen -más que en las necesidades que los afecta-. Esta idea simple cambia el punto de vista de la comunidad desde una perspectiva de déficit a una perspectiva de los recursos (Cruz y Giles, 2000, 32). En 


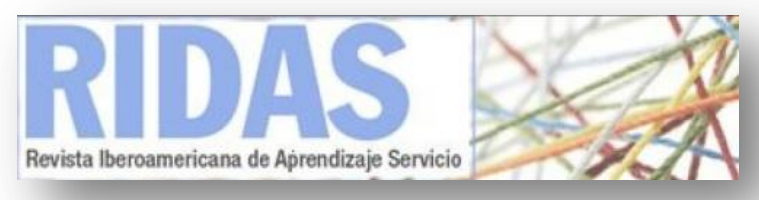

lugar de preguntar cuáles son las necesidades de la comunidad, preguntar por las potencialidades que tienen y cuáles pueden seguir desarrollándose.

\section{Cinco buenas prácticas para conformar una sociedad de aprendizaje servicio}

Comparto algunas buenas prácticas que las experiencias me han dado. Hablo en plural, porque justamente en mi calidad de asesor de experiencias universitarias, aquello que llama la atención a un docente u organización, no necesariamente llama la atención del otro. En ese sentido, estoy lejos de ofrecer la mejor estrategia de implementación de aprendizaje servicio -aunque parezca una mala carta de presentación- sino que a ayudar a mirar la situación de planificación e implementación a la luz de interrogantes que abran caminos de posibilidad.

1. Ajustar expectativas. Un proyecto excesivamente acotado puede no ser una contribución para la comunidad ni un espacio de aprendizaje significativo para los estudiantes. O bien, puede ser un proyecto demasiado grande, que genere ilusiones en la comunidad que luego no puedan cumplirse, generando frustración en todos los participantes. Difícilmente solucionemos el problema. Lo que podemos desarrollar es un avance a una problemática mayor. Es complejo que en un periodo de seis meses o un año, seamos capaces de resolver un desafío que para una comunidad le ha tomado años luchar. Sí podemos hacer una contribución, un avance sustantivo, e incluso plantear interrogantes que nunca se habían hecho. Eso resulta más sensato, más ajustado y con mayor posibilidad de éxito a la hora de evaluar el trabajo en su conjunto.

2. Establecer canales explícitos de comunicación. No basta con enviar un correo electrónico y esperar eternamente a que lo respondan. Anticiparse a esto, implica evaluar alternativas de comunicación y tiempos estimados de respuesta que favorezca la fluidez de los mensajes. Muchos proyectos se coordinan entre los directores y/o encargados de programas comunitarios saltándose a aquellos que ejecutan directamente las acciones. Aquí una recomendación muy pertinente, es definir desde un comienzo acuerdos, que estén escritos y que permitan darle seguimiento, incluso a las formas en que se acordaron las comunicaciones.

3. Generar alianzas interdisciplinarias. A veces nuestra ceguera disciplinaria no nos permita conectar oportunidades para que estudiantes de otras áreas puedan sumarse. Especialmente pensando en experiencias donde distintas disciplinas convergen a la vez en torno a una misma problemática.

4. Conociendo al otro veo mis puntos ciegos. A veces, desde el punto de vista de la Universidad, solo vemos falencias en las instituciones. Cuestionamos el tipo de intervención que desarrollan, el trato con las personas, los espacios físicos con los que 
cuentan, los recursos que poseen. Con esto, uno no reconoce las lógicas internas con las que actúan las instituciones. Observar las prácticas institucionales con detención y sin prejuicios, nos fuerza a desarrollar dispositivos de comprensión más complejos que no siempre ofrece la teoría- que permite un campo de comprensión mayor de la realidad: nos ayuda a entender como entienden otros, nos ayuda a entender por qué las intervenciones se desarrollan con determinados recursos. En definitiva, nos ayuda a reinterpretar los espacios de intervención.

5. No generar dependencia mutua. Generamos un trabajo que puede ser muy potente un año, y lo replicamos año a año, con la misma comunidad pero con estudiantes distintos, obligando a las organizaciones a recibirnos todo el tiempo. Tampoco es pertinente que las organizaciones dependan de la colaboración que año a año hacen los estudiantes sin generar autonomía. Aprendizaje servicio implica desde un comienzo construir un proyecto en conjunto y eso a veces nos cuesta. Desde la Universidad es muy fácil tomar un programa de curso y decir "somos capaces de hacer esto y es lo que le vamos a ofrecer a una escuela o una junta de vecinos", y eso es muy limitado si pensamos que las necesidades no solo son amplias sino que además complejas de abordar con una propuesta rígida que pudiera considerar el docente con sus estudiantes.

\section{Referencias bibliográficas}

Andreozzi, M. \& Nicastro, S. (2006). Asesoramiento pedagógico en acción. La novela del asesor. Buenos Aires: Editorial Paidós.

Cruz, N. \& Giles, D. (2000). Where's the Community in Service-Learning Research? Michigan Journal of Community Service Learning, Special Issue, 28-34. Chicago: Ebsco.

Eyler, J. \& Giles, D. (1999). Where's the learning in service-learning? San Francisco: The Jossey-Bass.

Furco, A. \& Billig, S. (Eds.) (2002). Service-Learning: the essence of the pedagogy. (Vol. 1, Advances in Service-Learning Research). Greenwich, CT: Information age.

Tapia, M. (2010). La propuesta pedagógica del "aprendizaje servicio": una perspectiva latinoamericana. Tzhoecoen, 3, 5, 28-43. Chiclayo: Universidad Señor de Sipán. 